\title{
On the coding of spatial information
}

\author{
JEAN M. MANDLER \\ University of California, San Diego, La Jolla, California 92093 \\ DALE SEEGMILLER \\ University of California, Berkeley, California 94720
}

and

JEANNE DAY

University of Illinois, Champaign, Illinois 61820

\begin{abstract}
Two experiments studied recall of objects and their locations in an intentional-incidental learning paradigm. When studying spatial information, the usual incidental condition is not truly incidental, because subjects often deliberately use locations to help organize objects for recall. Therefore, a true incidental task was devised in which neither objects nor locations were expected to be recalled and for which explicit encoding of locations was irrelevant. There was only a small loss in recall of objects or their locations in a true incidental condition. It was concluded that a great deal of location information is automatically coded into long-term memory storage in the sense that active processing is not required. The data were contrasted with incidental processing of other attributes, such as color. Although adults performed better than children, there were no age-related interactions, indicating similarity of functioning at all ages studied.
\end{abstract}

We have all had the experience of recalling an article from the morning newspaper and in unbidden fashion remembering where it was located on the page. Sometimes we can recall the location of a passage clearly even when we cannot remember its content. This kind of experience (studied by Rothkopf, 1971; Zechmeister, McKillip, Pasko, \& Bespalec, 1975) suggests that at least some spatial information is stored in long-term memory even when people are not attending to it. Although it is possible that we consciously attend to the location of the words on the page when we read, it seems unlikely. Certainly we rarely intend to remember this information. Many kinds of information about words or objects-their color, orientation, location-may be briefly registered, perhaps even attended, but are not necessarily coded into long-term storage.

When studying perception, we may ask whether information such as location is automatically encoded, that is, whether it is processed without intention or conscious awareness and does not interfere with other processing going on at the same time (Posner \& Snyder, 1975). In the study of memory, we may ask similar questions but they are apt to be posed slightly dif-

This research was supported in part by NIMH Research Grants MH 24492 and MH 15828. We would like to thank the San Diego, Unified School District and the teachers and students at Miramar Ranch Elementary School for their helpful cooperation. We also thank Nancy Weile, who helped in data collection. Requests for reprints should be sent to Jean M. Mandler, Department of Psychology C-009, University of California, San Diego, La Jolla, California 92093. ferently. When it is a question of what will be remembered, we usually ask whether the person intended to remember location and attended to it for that purpose, and if so, whether processing location interfered with other information which the person was also trying to remember. In both areas of research the term "automatic processing" has been used when no intention or interference is found (e.g., Light \& Berger, 1974; von Wright, Gebhard, \& Karttunen, 1975), although to avoid confusion we should note the slightly different usage in the two cases.

The present report addresses the question of whether locations of objects are automatically encoded in the sense of being remembered even when a person does not try to remember them, and whether a deliberate attempt to remember the locations of objects interferes with memory for the objects themselves. We know somewhat more about incidental processing of color than we do about spatial information. Light and Berger (1974) and Light, Berger, and Bardales (1975) have shown that both the color and case in which words are presented are better remembered in an intentional learning condition (i.e., subjects are told to remember both the words and their modality) than in an incidental learning condition (i.e., subjects are told only to remember the words). However, there is a trade-off between the two memory tasks, since more words are remembered in the incidental than in the intentional condition. Thus, remembering the color of an object as well as its identity is a dual-processing task, with cost associated with processing of both kinds of information. At the same time, color and case are recognized 
at slightly above chance levels in at least some of the incidental conditions, suggesting that some color information is stored without deliberate intent on the part of the subjects.

Results have been mixed in the few studies of incidental memory for location. Zechmeister et al. (1975) found no differences in accuracy of item or location recall in either intentional or incidental conditions. Thus, there was no trade-off when attempting to remember the two kinds of information. In both conditions memory for location was well above chance. von Wright et al. (1975) also found no differences between intentional and incidental recall of the locations of pictures, nor any trade-off between item and location recall in the two conditions. Recall of locations in both conditions was high.

Schulman (1973), however, found a partial tradeoff between word recognition and recall of location. Subjects in an intentional condition recognized fewer words than subjects in an incidental condition; however, they also recalled fewer locations. The latter is a surprising finding, but may have been due to the particular task Schulman used. Subjects were required to learn 100 words presented in 25 arrays of 4 , each word appearing at the four points of the compass. Recall of locations was not greatly above chance. Schulman suggested that subjects may have adopted an inefficient strategy in the intentional condition, attempting to recode the spatial information into compass labels and thus treating the task as a paired-associate one.

This possibility raises the more general problem of the kinds of strategies which are used in both intentional and incidental conditions to organize and rehearse the information that is to be remembered. In standard incidental conditions, when subjects are asked to remember objects they may deliberately use the "incidental" information as a mnemonic device. This is especially apt to be true of spatial information, which can form the basis of an efficient mnemonic system for remembering items (Bower, 1970; Yates, 1966). The information that is supposed to be incidental is deliberately attended to in the service of remembering the other information. Although subjects may not intend to remember location, its deliberate processing may produce that result. What is needed is a truly incidental task in which subjects do not attempt to process the incidental information in any active way.

The present experiments used an incidentalintentional paradigm, but with three conditions instead of the usual two. In the intentional condition, subjects were told to remember both objects and their locations. In the standard incidental condition, they were told only to remember the objects. Finally, a true incidental condition was added in which subjects were not given memory instructions but were told to price the individual objects in order to estimate the cost of the total array. It was assumed that the pricing instructions would require deliberate processing of item identity but that the location of individual items would be irrelevant to the task and thus would not be deliberately processed.

Experiment 1 used adults as subjects. Experiment 2 was a replication and extended the findings to children. There are two developmental questions. The first concerns intentional processing, that is, whether there are age differences in ability to remember spatial information when deliberately attempting to do so. Although there is an anecdotal suggestion that children are very good at remembering locations (the game of Concentration is thought to be popular among children because they can beat adults at it), the small amount of experimental evidence available (e.g., Finkel, 1973) suggests that, as in most other deliberate memory tasks, adults are more efficient encoders than children. The second question concerns incidental processing, that is, whether children store spatial information in tasks in which their attention is not deliberately directed to it.

Acredelo, Pick, and Olsen (1975) found that children from 3 to 8 years of age were able to locate a place where a given event had occurred more accurately under intentional recall conditions than under incidental conditions. On the other hand, von Wright et al. (1975), using a design similar to Schulman's (1973), found no differences between intentional and incidental recall of the locations of pictures at any age from 5 years to adult. They concluded that location is encoded au tomatically, even by young children. There were many differences between these two studies, such as the use of events vs pictures. In particular, it is not known what the children were attending to in the incidental condition in the Acredelo et al. experiment; von Wright et al. assumed that subjects in the incidental condition were concentrating on memorizing the pictures. Again, it appears important to control degree and focus of attention in incidental and intentional conditions.

Since children may differ from adults in their use of spatial location as a deliberate strategy to remember items, the present experiments used the additional true incidental condition described earlier in which location is irrelevant to the task. In addition, every item was in a unique location so as to eliminate possible interference caused by many items occurring in the same location. A method of presentation was used which was designed to minimize recoding of spatial information into another mode, such as the verbal associations which Schulman's (1973) subjects may have used. Pilot work indicated that, at least with adults, this condition is not easy to attain. We originally used 25 randomly chosen nouns in a 5 by 5 array, only to discover that when told to remember the words, subjects created sentences for each row. When asked to recall locations of the words, the order of the words in the sentences provided location information independently of any spatial memory. 
For this reason we abandoned words as stimuli and regular matrices as arrays and worked instead with real objects placed in seemingly random arrays.

\section{EXPERIMENT 1}

\section{Method}

Subjects. Subjects were 96 undergraduates from the University of California, San Diego, who participated in the experiment as a course requirement in a lower division psychology class.

Materials. Sixteen small toys were used as stimuli. They were chosen to be easily identifiable and to be all roughly in the same price range. No more than two items belonged to the same conceptual category (animals, vehicles, etc.).

A matrix of 36 locations (each approximately $7.6 \mathrm{~cm}$ square) was generated in six rows and six columns within a $46-\mathrm{cm}$-square area on a table top. From this set of locations, 16 locations were randomly selected, with the restriction that no more than 4 occur in any given row and that 4 locations occur within each quadrant of the matrix. The 16 items were then randomly assigned to the 16 locations and were varied from subject to subject so that each item occupied each location in the matrix equally often. Every different order was tested once in each cell of the design. An object's orientation with respect to the array was maintained throughout the experiment. A 46-cmsquare clear plastic sheet was made to correspond to the matrix and $2.5-\mathrm{cm}$ blue dots were placed in the center of the 16 object locations. This sheet was used to designate the locations to which subjects were to assign objects when they reconstructed the array.

Questionnaires were constructed to assess each subject's strategies for studying and recalling items and locations. The questionnaire was also used to determine the extent to which subjects understood and followed instructions for each condition. One subject in an incidental condition who indicated that he had guessed he would be asked to recall locations was replaced.

Design. Sixteen subjects were randomly assigned to each cell of a matrix consisting of three types of instruction (intentional, standard incidental, and true incidental) and two methods of reconstruction (objects remain, objects removed). All subjects were asked to study the array, and afterwards first to recall the objects, then to reconstruct the array by placing all objects in their correct locations.

In the intentional condition, subjects were told to study the items so that they could later recall them and place them in their correct locations. In the standard incidental condition, subjects were told only to study the items so that they could later recall them. In the true incidental condition, subjects were told to study the group of items and determine a reasonable estimate of the cost of the entire group.

After studying the array, subjects were asked to recall as many objects as they could. Following this task all objects were presented to the subjects and they were asked to place them in their correct locations, using the plastic sheet with the marked locations. Two types of reconstruction condition were used. In the remain condition, once an object had been placed on the sheet it remained in that location until the task was completed. In the remove condition, each object was removed from view after it had been placed, and the location was marked with a piece of paper to indicate that it had already been selected. These two conditions varied the degree of memorial support provided by the objects themselves during the reconstruction task.

Procedure. Subjects were tested individually. Each subject was read the appropriate instructions. They were then allowed to study the array for $40 \mathrm{sec}$. All subjects were instructed to be sure to look at all of the objects. After studying the array, subjects went into another room and wrote down as many of the items as they could recall in a 3-min period. The incidental group also wrote down their estimated price of the array. Subjects were then told that they were to reconstruct the array that they had studied. They returned to the table where they had studied the array and were shown the plastic sheet with the locations marked on it. The objects were all placed in front of the subject, who was allowed to determine the order in which they were placed on the sheet. Subjects were told to make their placements one at a time and to state their confidence as to how sure they were that a particular location was correct $(1=$ not sure, 2 = fairly sure, 3 = very sure). Subjects were told that once they placed an item in a location, they could not change it. Order of placement, confidence ratings, and items placed in each location were recorded. Finally, subjects completed the questionnaire and were debriefed.

\section{Results}

Mean number of objects recalled and mean number of objects placed in the correct locations are shown in the first two rows of Table 1. The third row shows the mean number of objects that were both recalled and correctly placed. An analysis of variance of object recall showed that instructional condition was a significant source of variance $[F(2,90)=6.56, \quad p<.01, \quad M S e=4.16]$. Newman-Keuls tests indicated that the intentional and standard incidental conditions did not differ significantly from each other, but both were superior to the true incidental condition ( $\mathrm{p}<.05$ and .01 , respectively). The loss in number of objects recalled in the true incidental condition compared to the mean of the other two conditions was 1.4 items.

An analysis of variance of number of objects placed in the correct locations showed that the effect of instructions, although in the same direction as for object recall, did not reach significance $[F(2,90)=2.86$, $\mathrm{p}<.10, \mathrm{MSe}=3.14]$. The loss in the true incidental condition compared to the mean of the other two conditions was 1.8 locations. The remain vs remove manipulation was not a significant source of variance.

An analysis of variance comparing number of objects recalled, number of objects correctly placed, and number of objects correct on both measures $[F(3,90)=$ $139.07, \mathrm{p}<.001, \mathrm{MSe}=2.79$ ] showed these three measures to differ significantly. Subjects could recall a mean of two more objects than locations. Another two items were lost when the scoring requirement of being correct on both recall and location was imposed. There was no interaction with instructional condition,

Table 1

Mean Number of Objects Recalled, Objects Correctly Located, and Objects Both Recalled and Located (Total Possible $=16$ )

\begin{tabular}{lcccc} 
& \multicolumn{3}{c}{ Condition } \\
\cline { 2 - 4 } & $\begin{array}{c}\text { Standard } \\
\text { Inten- } \\
\text { tional }\end{array}$ & $\begin{array}{c}\text { True } \\
\text { dental }\end{array}$ & $\begin{array}{c}\text { Inci- } \\
\text { dental }\end{array}$ & Mean \\
\hline Objects & 10.3 & 11.2 & 9.3 & 10.3 \\
Locations & 8.7 & 9.1 & 7.1 & 8.3 \\
Both Correct & 6.8 & 7.3 & 4.8 & 6.3 \\
\hline
\end{tabular}


indicating that the differences were approximately the same in all three conditions (see Table 1).

In all conditions the probability of correctly locating objects was greatly above chance. The mean of the chance distribution for placing items correctly without replacement in an array of 16 items is one, with a likelihood of occurrence of .37 (Chapman, 1934). The probability of placing as many as five items correctly by chance is less than .01 . An object was more likely to be correctly placed if it had been recalled (.53) than if it had not been recalled (.39). Although this difference is highly significant $[F(1,90) 14.36, p<.001, \quad \mathrm{MSe}=$ $.06]$, the likelihood of locating a nonrecalled object is still far above chance. There were no significant effects of instructional conditions on the two conditional probabilities, indicating the same degree of relationship between the two kinds of information in intentional and incidental conditions.

Another way to assess the degree of association between object recall and correct placement is to compare the obtained proportion of objects both recalled and correctly placed with the expected proportion if the two kinds of information were independently processed. On the assumption of independence the expected proportion is .33; the obtained proportion was .39 $(p<.001)$. The differences between expected and obtained proportions were equivalent in all three instructional conditions. Although reliable, the differences between each of the expected and obtained proportions were small, indicating that there is only a slight degree of association between recall of an object's identity and its location.

An analysis of variance of correct placements in the four quadrants of the array showed significant differences $[F(3,270)=5.59, p<.01, \mathrm{MSe}=1.07]$. Objects were more likely to be correctly located if they had appeared in the left half of the array; in particular, performance was poorest in the upper right quadrant. This finding suggests that subjects spent more time processing the left half and bottom of the array. However, such differences were not reflected in object recall; objects were equally likely to be recalled from all four quadrants. There were no significant effects of instructional condition on quadrant placement.

Instructions did not significantly affect confidence in correctness of locations. Subjects were more confident of their placements in the remain condition than in the remove condition $[\mathrm{F}(1,90)=4.13, \mathrm{p}<.05, \mathrm{MSe}=.62]$; however, as noted earlier, there was no difference in accuracy in these two conditions.

In summary, the data from this experiment indicate that almost as much spatial information is retained when it has not been attended as when it has been deliberately processed. There was only a slight (and in this experiment, nonsignificant) loss in number of locations retrieved when a true incidental condition was used in which processing of locations was irrelevant. The loss in recall of locations was even smaller than the loss in recall of the objects themselves, although the requirements of the pricing task would seem to require active processing of object identities.

The lack of significant differences on any measure between the intentional and the standard incidental condition indicates that not telling subjects they will be tested on spatial information does not mean that they will not attend to it. This hypothesis was confirmed by the questionnaire data. Seventy-two percent of the subjects in the intentional condition reported using some type of spatial strategy. However, $50 \%$ of the subjects in the standard incidental condition also reported using location as a mnemonic aid for object recall. These data suggest that spatial location is frequently used by subjects to help them remember objects. Thus, the standardly used incidental condition may foster intentional processing of the "incidental" information. In contrast, only $9 \%$ of the subjects in the true incidental condition reported studying objects by rows or in spatial groups in their attempt to determine the overall price of the array, and all reported no expectation they would be asked to recall locations.

\section{EXPERIMENT 2}

\section{Method}

Subjects. There were 192 subjects in this experiment, 48 each from kindergarten, third grade, six th grade, and university. The children were from a single public school in San Diego, and the adults were from the same population as that described in Experiment 1.

Materials. All materials were identical to those in Experiment 1 , except several of the toy objects which might have been unfamiliar to kindergarten children were replaced with more familiar items. No questionnaires were used in this experiment.

Design. The design was essentially the same as in Experiment 1 . Sixteen subjects from each grade level were randomly assigned to the three instructional conditions. Since there were no differences in accuracy in Experiment 1 due to the remain vs remove conditions, and since subjects had indicated they were more confident of their placements in the remain condition, only that condition was used in this experiment.

Procedure. Subjects were tested individually; children were tested in a laboratory van at their school. Subjects were allowed $60 \mathrm{sec}$ to study the array, instead of the $40 \mathrm{sec}$ used in Experiment 1. After study, they turned around and were asked to recall the objects verbally instead of writing them down. No confidence judgments were required. All other details of procedure were the same as in Experiment 1.

\section{Results}

Mean number of objects recalled, located, and correct on both recall and location under the three instructional conditions for each of the four age groups is shown in Figure 1. In Experiment 1, more objects were recalled than were correctly located. In this experiment there was no main effect for objects vs locations $[F(1,180)=$ $2.35, \mathrm{p}>.10]$. There was, however, an interaction with grade level $[\mathrm{F}(3,180)=3.10, \mathrm{p}<.05, \mathrm{MSe}=5.13]$. A breakdown of this interaction showed that third and sixth graders correctly placed more objects than 


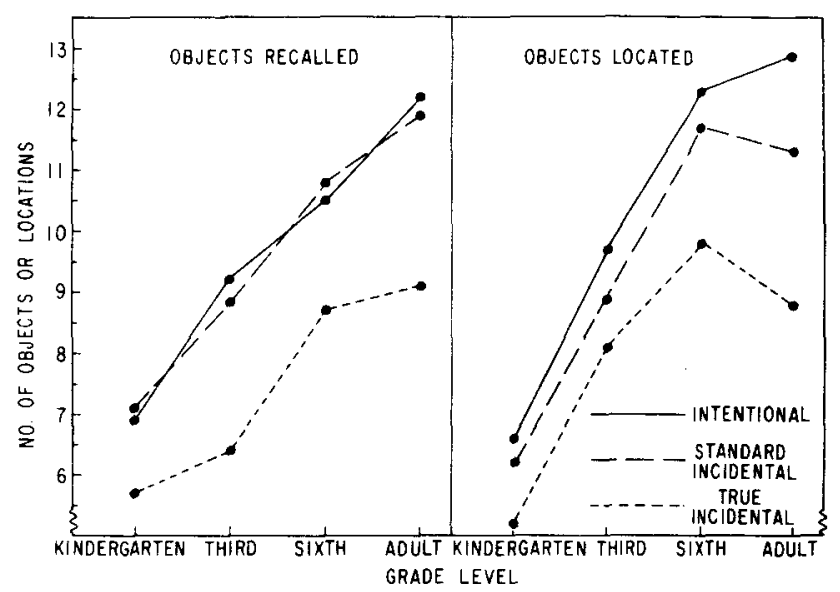

Figure 1. Number of objects recalled and number of objects correctly located by children and adults in three instructional conditions.

they recalled, significantly so for the sixth graders $(p<.01)$. There were no differences between these measures for either kindergarten children or adults.

The analysis showed a significant effect of age on recall $[F(3,180)=47.02, \quad p<.001, \quad M S e=9.82]$. Newman-Keuls tests indicated that, for both objects and locations, kindergarten children scored lower than the third grade, and the third grade lower than the sixth grade and adults; the latter two groups did not differ significantly from each other. Instructional conditions also differed significantly $[F(2,80)=19.71$, $\mathrm{p}<.001, \mathrm{MSe}=9.82]$. Intentional and standard incidental conditions did not significantly differ, but both were better than the true incidental condition for both objects and locations $(p<.01)$. On the average, 2.2 fewer objects and 2.0 fewer locations were recalled in the true incidental condition than in the other two conditions.

The interaction of Instructional Condition by Grade Level was not significant, indicating that subjects of all ages performed in a similar fashion under the different instructions. It should be noted, however, that the decline in performance in the true incidental condition was significant only for adults $(p<.01)$.

Similar results were found for the conditional probabilities of correctly locating objects given that they had been or had not been recalled. These data are shown in Figure 2. Again there was a main effect of grade level $[F(3,180)=17.72, p<.001, \mathrm{MSe}=.09]$. Kindergarten children performed more poorly than third graders $(p<.01)$. Third graders did not differ significantly from adults, but were poorer than sixth graders $(\mathrm{p}<.01)$. In this experiment, as opposed to the previous one, there was a significant main effect of instructional condition; the probabilities of locating an object correctly, whether recalled or not, were lower in the true incidental condition than in the other two instructional conditions $[\mathrm{F} 2,180)=6.42, \mathrm{p}<.01, \mathrm{MSe}=.09]$.
The interaction of Grade Level by Instruction Condition was not significant, although as in the previous analysis, the decline in performance in the true incidental condition was only significant for the adults $(\mathrm{p}<.01)$.

As in Experiment 1 , the probability that an object was correctly placed given that it had been recalled (.67) was higher than the probability that an object was placed given that it had not been recalled (.49) $[\mathrm{F}(1,180)=94.05, \mathrm{p}<.001, \mathrm{MSe}=.03]$. The obtained probability of both recalling and correctly locating a given object ( .39$)$ was significantly greater than expected by chance if the two kinds of information were independent $(.33)(\mathrm{p}<.001)$. The differences between the two probabilities were equivalent at each age level and instructional condition. Again, it should be noted that although significant, the difference between these two probabilities (which were the same as in Experiment 1), was unimpressive. Thus, only a small degree of association between recall of objects and their locations was found.

The probabilities of locating objects given recall or no recall were higher than in Experiment 1 , presumably due to the longer study time used in Experiment 2. A comparison of adult performance (in the remain conditions) across the two experiments showed that the increase in study time did not significantly increase the number of objects recalled $[F(1,90)=2.74, p>.10]$. It did, however, result in more objects being correctly placed $[F(1,90)=12.00, p<.001, \mathrm{MSe}=12.09]$. This increase was primarily due to the intentional condition, in which adults correctly placed four more objects than in Experiment 1. There was no significant improvement in the other two conditions.

Experiment 2 showed the same pattern of correct placements in the four quadrants of the array as found in Experiment $1[\mathrm{~F}(3,540)=14.18, \mathrm{p}<.001, \mathrm{MSe}=$ $1.13]$. Objects were more likely to be correctly placed

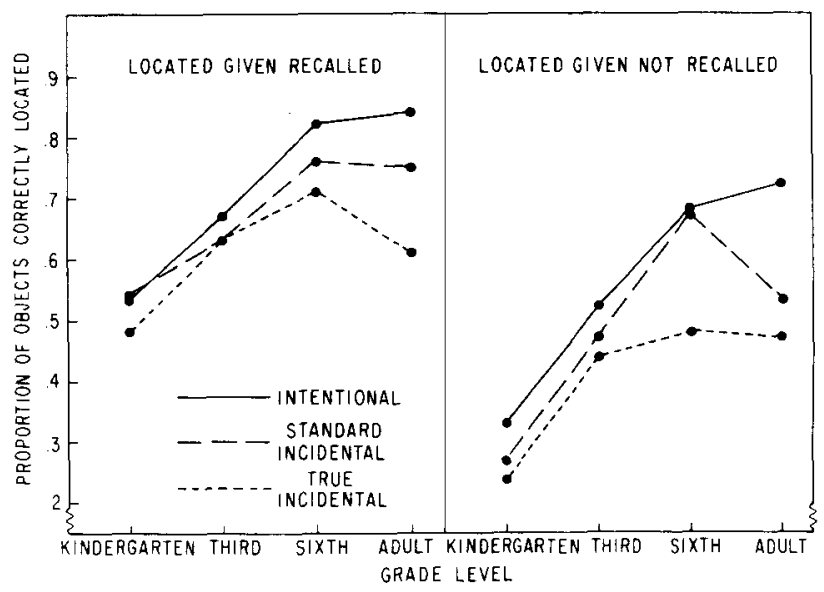

Figure 2. The probabilities of lacpting an object given it had been recalled and given that it had not been recalled, for children and adults in three instructional conditions. 
if they had appeared in the left half of the array and performance was poorest in the upper right quadrant. Again the effect of position in the array on likelihood of objects being recalled was not significant.

In summary, there was good agreement between the two experiments. In particular, the lack of significant interactions between instructions and grade level suggests similar modes of functioning under the various instructions across the ages studied. Although the data suggested that adults performed relatively more poorly under true incidental instructions than did children, the lack of overall age-related interactions indicates caution in interpreting this finding.

\section{DISCUSSION}

The data from these experiments demonstrate that a great deal of spatial information is available for retrieval without attention having been directed to it. In the first experiment the loss in location information retrieved in the true incidental learning condition compared to the intentional learning condition was only $18 \%$. In the second experiment, more study time was given, and subjects in the intentional condition, not surprisingly, retrieved more locations. Adult subjects in the true incidental condition retrieved $32 \%$ fewer locations, and children 19\% fewer.

In the first experiment, more objects were recalled than object locations. When somewhat longer study time was given, there were no overall differences in recall of the two types of information, although sixth graders were better at object locations. The loss in retrievability of object identities in the true incidental condition was slightly less than that found for object locations. In the first experiment there was a $10 \%$ loss in object recall in the true incidental condition, compared to the intentional condition; in the second experiment the loss was $25 \%$ for adults and $21 \%$ for children.

There was no cost to object recall when subjects were required to remember locations as well. The data suggest that the processing of an object's location may be qualitatively different than processing of other attributes, such as color. Light and Berger (1974) and Light et al. (1975) have shown that, when subjects are required to remember the color of items, there is a trade-off in which attention to color results in lesser processing of the items themselves. However, the lack of any significant differences in the intentional and standard incidental conditions, combined with subjects' reported use of locations to help memorize objects, suggests that, at least for spatial information, the typically used incidental condition is not adequate to assess the cost of remembering two kinds of information. If one kind of information is used to encode another, then a trade-off would not be expected.

If this is the case, then the use of a truly incidental condition, such as a pricing task, is essential to assess the extent to which various kinds of information are automatically coded into long-term storage. An interesting comparison can be made between the standard incidental and true incidental conditions. In the former, subjects did not intend to remember locations but actively processed them to help organize objects for recall; the result was that they remembered as much as when they intended to remember both kinds of information. In the true incidental condition, subjects did not intend to remember either kind of information, but presumably actively processed object identities in order to price them, while ignoring their locations. Nevertheless, loss in retrievability of locations was no greater than for objects. Thus, active processing does not seem to be required for spatial information to be encoded into long-term storage.

Finally, the present data indicate a great deal of similarity in handling object and location information throughout the developmental span studied. In the first instance it is of interest that the same set size (16 objects and locations) could be used for kindergarten children as for adults. It is true that kindergarten children remembered less of both kinds of information than adults, but in pilot work it was found inadvisable to use a smaller set size, since some young children reached $100 \%$ performance on locations in arrays of 12 objects. This finding may be contrasted with typical studies of memory for word lists, in which it is usually necessary to present fewer words to children than to adults in order to avoid overloading the children's processing capacity (e.g., Worden, 1974).

In general, the present data are consistent with those of von Wright et al. (1975), in that children did no worse in incidental conditions relative to intentional conditions than adults; in fact, they did slightly better. Only adults showed a significant loss in the true incidental condition. However, we should not conclude that children are more automatic encoders than adults. It was not considered productive to question children about their strategies in organizing information. However, the pattern of results in the intentional and standard incidental conditions was so similar for all age groups that it seems reasonable to assume children were also using spatial location in their attempt to remember the objects. In the pricing situation, however, it is possible that children actively processed location information more than did the adults. Although it will be difficult to assess, we still need to determine whether there is a wider scattering of attention in younger children to various attributes of the array, accompanied by less concentration on the assigned task. In absolute terms, of course, adults were better in all types of conditions. The data suggest that, despite rumors to the contrary, you have a good chance of beating your children at Concentration.

\section{REFERENCES}

Acredelo, L. P., Pick, H. L., JR.. \& Olsen, M. G. Environmental differentiation and familiarity as 
determinants of children's memory for spatial location. Developmental Psychology, 1975, 11, 495-501.

Bower. G. H. Analysis of a mnemonic device. American Scientist. 1970, 58, 496-510.

Chapman. D. W. The statistics of the method of correct matchings. American Journal of Psychology, 1934, 46. 287-298.

FinkEL. D. L. A developmental comparison of the processing of two types of visual information. Journal of Experimental Child Psichology, 1973, 16, 250-266.

Light. L. L.. \& BERGER, D. E. Memory for modality: Withinmodality discrimination is not automatic. Journal of Experimental Psychology, 1974, 103, 854-860.

Light. L. L., Berger, D. E.. \& Barbales, M. Trade-off between memory for verbal items and their visual attributes. Journal of Experimental Psychology Human Learning and Memon. 1975. 104, 188-193.

Posner. M. I., \& SNyder, C. R. R. Attention and cognitive control. In R. L. Solso (Ed.), Information processing and cognition: The Loyola symposium. Hillsdale. N.J: Lawrence Erlbaum. 1975.
Rотнкорғ, E. Z. Incidental memory for location of information in text. Journal of Verbal Leaming and Verbal Behavior. 1971, 10, 608-613.

Schulman, A. I. Recognition memory and the recall of spatial location. Memory \& Cognition. 1973, 1. 256-260.

von Wright, J. M., Gebhard, P., \& Kartiunen, M. A developmental study of the recall of spatial location. Journal of Experimental Child Psychology, 1975, 20, 181-190.

WORDEN, P. E. The development of the category-recall function under three retrieval conditions. Child Development, 1974, 45, 1054-1059.

YATES, F. A. The art of memory. Chicago, Ill: University of Chicago Press, 1966.

Zechmeister, E. B., McKillip, J., Pasko. S., \& Bespalec, D. Visual memory for place on the page. Journal of General Psychology. 1975. 92. 43-52.

(Received for publication June 30, 1976: revision accepted November $1,1976$. 